\title{
Bayesian Network Assessment Method for Civil Aviation Safety Based on Flight Delays
}

\author{
Huawei Wang ${ }^{1}$ and Jun Gao \\ ${ }^{1}$ College of Civil Aviation, Nanjing University of Aeronautics and Astronautics, Nanjing 210016, China \\ ${ }^{2}$ Department of Management, Shijiazhuang Mechanical Engineering College, Shijiazhuang 050003, China \\ Correspondence should be addressed to Huawei Wang; huawei678@163.com
}

Received 27 October 2013; Revised 28 November 2013; Accepted 29 November 2013

Academic Editor: Fang Zong

Copyright (c) $2013 \mathrm{H}$. Wang and J. Gao. This is an open access article distributed under the Creative Commons Attribution License, which permits unrestricted use, distribution, and reproduction in any medium, provided the original work is properly cited.

\begin{abstract}
Flight delays and safety are the principal contradictions in the sound development of civil aviation. Flight delays often come up and induce civil aviation safety risk simultaneously. Based on flight delays, the random characteristics of civil aviation safety risk are analyzed. Flight delays have been deemed to a potential safety hazard. The change rules and characteristics of civil aviation safety risk based on flight delays have been analyzed. Bayesian networks (BN) have been used to build the aviation operation safety assessment model based on flight delay. The structure and parameters learning of the model have been researched. By using BN model, some airline in China has been selected to assess safety risk of civil aviation. The civil aviation safety risk of BN model has been assessed by GeNIe software. The research results show that flight delay, which increases the safety risk of civil aviation, can be seen as incremental safety risk. The effectiveness and correctness of the model have been tested and verified.
\end{abstract}

\section{Introduction}

The rapid increase of flight delays has already become a prominent problem in the development of civil aviation in China. It not only affects the service quality and economic benefit of civil aviation, but also reduces the civil aviation safety level. The flight delays have attracted public attention and become one of the key factors in impeding the development of civil aviation in China.

The close relationship between flight delays and safety risk is the two principal contradictions, which seriously affects the development of civil aviation in China. The complicated relation is principally due to the following reasons. Firstly, the operation and management subject of flight regularity and civil aviation safety are coincident. Secondly, the factors, such as resource shortage and operation management behavior, can give rise to the flight delays and safety operation simultaneously. Thirdly, the environmental factors, such as severe weather and human disturbance, result in not only flight delays but also safety risk. At the same time, flight delays cannot only result in potential safety risk of civil aviation but also come up simultaneously. Some specialists in civil aviation have realized that flight delays are an important potential risk in the management of civil aviation safety. If the safety risk, which is concurrent and induced, is not controlled in time, the aviation accident and grievous potential accident will take place under certain conditions. The aviation accident will bring about heavy losses.

The civil aviation safety level in China is in the state of steady improvement. Now China has already become one of the countries, who have high civil aviation safety level in the world. However, the number of delay flights in China is always very high. In 2012, there were 2,502,000 scheduled flights, which include 1,872,000 regular flights and 630,000 irregular flights, and the average flight punctuality rate is $75.69 \%$. The flight delays are caused $38.5 \%$ by airlines, $25.0 \%$ by flow control, and $21.6 \%$ by weather, so it is very severe in the development of civil aviation. The main reason that can affect civil aviation safety and flight delays is that the attributes of flight delays are subject to dominant indicators and the attributes of civil safety are subject to potential indicators. Potential safety risk exists and is in the high state, which will pose a threat to the safety of civil aviation. It may lead to the fluctuation of safety level of civil aviation and even 
worse the situation of civil aviation. Therefore, it is necessary to analyze the effect of flight delays on the safety of civil aviation in detail, assess the safety level of civil aviation based on flight delays, and provide measures and suggestions for the collaborative management of flight delays and the civil aviation safety.

Some scholars had already researched the related problems on flight delays and civil aviation safety. Airline maintenance operation affects not only flight delays but also civil aviation safety. Sachon and Paté-Cornell [1] built a probabilistic risk analysis model, represented by an influence diagram, to quantify the effect of airline's maintenance policy on flight delay, cancellation, and in-flight safety. McCrea et al. [2] made a novel severe-model paradigm to be applied for the context of a large scale in specified probability threshold when encountering severe weather, subject to collision safety, airline equity, and sector workload considerations. More research is to study flight delay and the aviation safety problem, respectively. In the respect of flight delay, Zheng et al. [3] calculated the time series of after-affect delay spread time using delay time distribution of the flights assigned gate in airport and random delay in flights. Wong and Tsai [4] used coproportional hazards model to build departure and arrival delay models that show how flight delay propagation can be formulated through repeated chain effects in aircraft rotations. Yan and Tang [5] developed a heuristic approach embedded in a framework designed to help the airport authorities make airport gate assignments that are sensitive to stochastic flight delays. Pyrgiotis et al. [6] developed an analytical queuing and network decomposition model to study the complex phenomenon of the propagation of delays within a large network of major airports, which has been used to compute the delay due to local congestion at individual airports and captures the "ripple effect" that leads to propagation of these delays. Santos and Robin [7] found that four significant variables in explaining delays at European airports were market concentration, slot coordination, hub airports, and hub airlines using flight data for the period 2000-2004. Using longitudinal data from a major airline and conditional difference-in-differences technique, Ferrer et al. [8] analyzed the effects of flight delay on passengers' future purchasing behavior. To develop a decision-support tool for air traffic control, presented an algorithm for optimal arrival flight sequencing and spacing in near-terminal, which used discrete delay times as optimization variables.

In the field of civil aviation safety, some scholars utilized data on both accident and safety indicators. Shyur [9] presented a specified proportional hazard model considering the baseline hazard function as a quadratic spline function, which had investigated and demonstrated its applicability in aviation risk assessment. Janic [10] described the main cause of aircraft accident, proposed a methodology for quantifying risk and safety, and offered an assessment of risk and safety in civil aviation. Chen et al. [11] ranked the significant threats and human errors affecting aviation safety and used the analytical hierarchy process to calculate the weigh for each criterion which were than ranked in order of importance. Marais and Robichaud [12] investigated and quantified the contribution of maintenance, both in terms of frequency and severity, to passenger airline risk by analyzing three different sources of data from 1999 to 2008. Brooker [13] examined the ability of Bayesian belief network-based techniques to make accurate aviation risk predictions. Hadjimichael [14] developed a flight operation risk assessment system (FORAS), which was a risk modeling methodology representing risk factors and their interrelationships as a fuzzy expert system. A FORAS risk model provided a quantitative risk index representing an estimate of cumulative effects of potential hazards on a single flight operation. Lee [15] developed a quantitative model for assessing aviation risk factors as a means of increasing the effectiveness of safety risk management system by integrating the fuzzy linguistic scale method, failure mode, effects and criticality analysis principle, and as low as reasonably practicable approach. Rose [16] studied the limitations and problems of trying to measure safety and operational risk and presented useful metrics from incident reporting data. Oster Jr. et al. [17] brought forward that the next generation of safety challenge now required development and understanding of new forms of data to improve safety in other segments of commercial aviation, moving from a reactive, incident-based approach toward a more proactive, predictive, and system-based approach.

From the above research literature, we understand that there is little study in the systematic analysis of flight delays, which have an effect on civil aviation safety and safety assessment.

The complexity of civil aviation safety risk assessment based on flight delays shows in the following aspects.

(i) There are many factors that affect flight delays and civil aviation safety risk. Coupling with the interaction between flight delays and civil aviation increases the difficulty of analysis.

(ii) The mechanism of flight delays, which can erupt simultaneously and induce civil aviation safety, remains to be further analyzed in detail.

(iii) The rate of civil aviation accident is low. But the increase of potential safety hazard and the deviation of safety state may not arise in the shape of accident, accident threat, or unsafe event immediately.

(iv) It is difficult to collect the direct statistics of the relevant factors. So, it is often completed with the help of subjective information by experts' experience.

Therefore, the difference between aviation safety risk based on flight delays and general aviation risk assessment can be concluded.

(i) Change of Assessment Elements. The general civil aviation safety assessment only focuses on the elements of safety itself. The safety assessment of civil aviation based on flight delays is one of the assessment elements, which not only reflects the value change of safety elements, but also embodies the relationships between the civil aviation safety system and flight delay system.

(ii) The Difference of Definition the Analysis Scope. Because both the flight delays system and civil aviation safety system 
are big systems, it is difficult or insoluble for civil aviation safety assessment based on flight delays if all the elements of flight delay system and civil aviation system are considered. So, emphasis is given to civil aviation safety elements associated with flight delays, which is different from general civil aviation safety assessment.

(iii) The Difference of Risk Evolution. The elements of flight delay system are dynamic, which is real-time change. The risk elements of civil aviation safety system are relatively stable. So, the civil aviation safety assessment based on flight delays is dynamic, while the general civil aviation safety assessment is relatively stable. follows.

The main contribution of the paper can be concluded as

(i) The composition of civil safety risk based on flight delays has been analyzed systematically, which includes the aggregation and the transmission of civil aviation safety risk;

(ii) The change rules of civil aviation safety risk based on flight delays have been analyzed, which include the increase of potential safety risks leading to civil aviation safety risk, the common causes of safety risk and flight delays leading to aviation safety risk, and flight delays leading to the increase in civil aviation safety risk degree as an inducement;

(iii) The safety assessment model of civil aviation by $\mathrm{BN}$, which considered the composition of civil safety risk based on flight delays, the randomness of civil aviation safety risk variation, the change rules of civil aviation safety risk based on flight delays.

Therefore, according to the characteristics of civil aviation, the paper presents an assessment model of civil aviation safety risk based on flight delays. The remaining part of the paper is organized as follows. Section 2 introduces the frame structure of algorithm. Section 3 analyzes the characteristics and change regulation of civil aviation safety risk based on flight delay. Section 4 builds an assessment model of civil aviation operation safety risk by the Bayesian network. Section 5 uses example to validate the effectiveness of the proposed method. Finally, some concluding remarks are made.

\section{The Risk Assessment Framework of Civil Aviation Safety Based on Flight Delays}

There are three criteria in building risk assessment framework of civil aviation safety in the paper.

(i) Considering the concurrence of subjective and objective data in risk assessment of civil aviation safety, the mechanism of action and dynamic change reflected by some factors in civil aviation system operation should be considered in building the model.

(ii) Flights delay should be seen as an incremental risk and be analyzed based on the risk analysis of civil aviation safety.

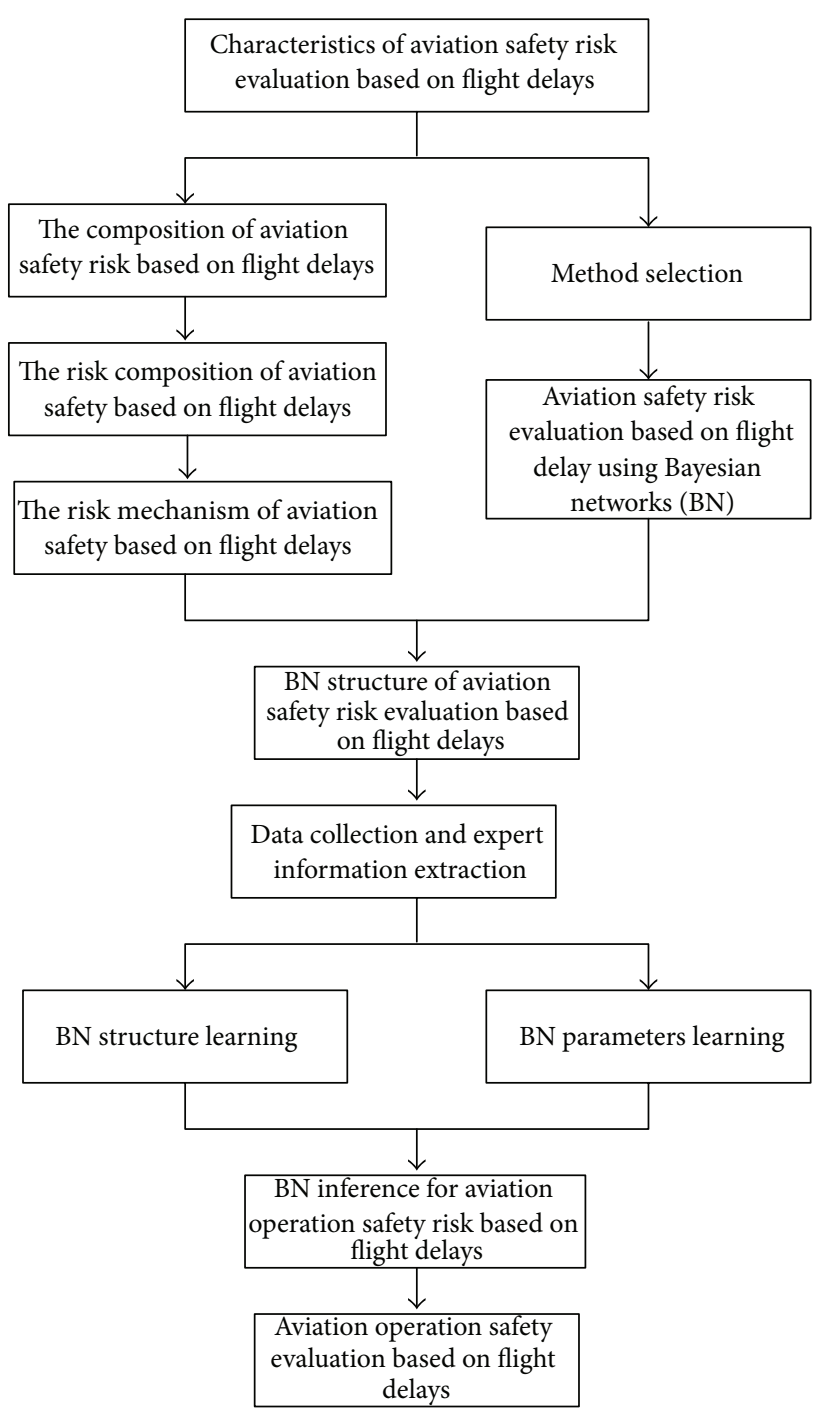

FIGURE 1: The diagram of civil aviation safety risk assessment based on flight delays.

(iii) The action mechanism and evolution law between civil aviation safety risk, which is induced by flight delays, and civil aviation operation system itself should be analyzed.

Figure 1 shows the diagram of civil aviation safety risk assessment based on flight delays.

\section{The Civil Aviation Safety Risk Based on Flight Delays}

\subsection{The Composition of Civil Aviation Safety Risk Based on Flight Delays}

(i) The Aggregation of Civil Aviation Safety Risk. The aggregation of civil aviation safety risk means that the actual risk of civil aviation safety consists of coinstantaneous and induced risks under flight delays. There are three main types of risk: 


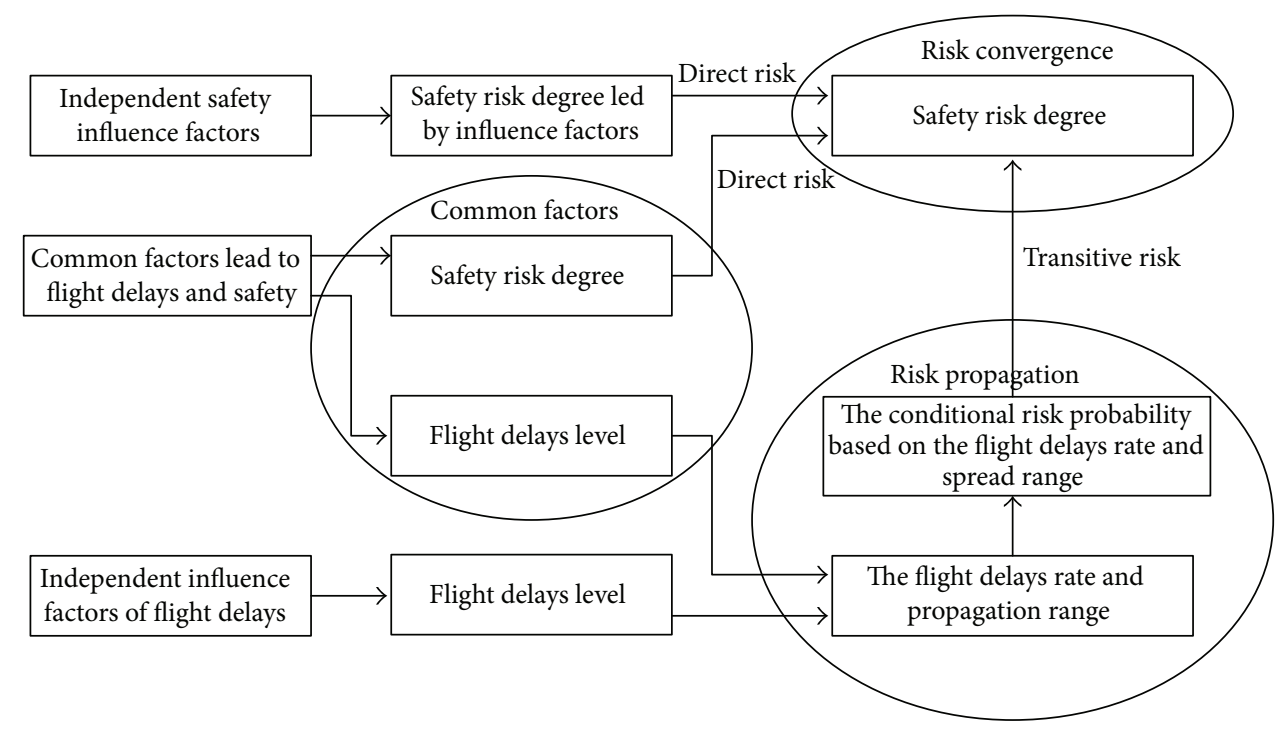

FIGURE 2: The entire constitute of civil aviation safety risk.

one is caused by the independent influence factors of civil aviation safety; the second is caused by the common factors which lead to flight delays and civil aviation safety risk; the third is caused by independent influence factors of flight delays.

(ii) The Transmission of Civil Aviation Safety Risk. The transmission of risk is from two aspects: one is the variation of the probability distribution of civil aviation safety risk according to different flight delays; the other is that the surging number of flight delay is caused by flight delays accumulation. The incensement of flight delays rate leads to the accumulation of civil aviation safety risk. Obviously, the civil aviation safety risk induced by flight delays changes with the rate and spread range of flight delays.

The entire constitute of civil aviation safety risk is shown in Figure 2.

\subsection{The Randomness of Civil Aviation Safety Risk Variation.} The civil aviation safety risk based on flight delays is random. The randomness is reflected as follows.

(i) Because the aviation accident is random, the civil aviation safety risk assessment is supposed to obey statistical probability distribution.

(ii) The appearance of flight delays is occasional. Although flight delays can be controlled to some extent, some flight delays caused by bad weather are generally unforeseeable and unavoidable. Therefore, some factors causing flight delay are random.

(iii) The occurrence of potential safety hazard factor is random. The potential safety hazard often appears as hidden factor, which is difficult to detect and will lead to safety risk when it is transformed into the dominant factor under some conditions. (iv) As a special safety risk, the influence of flight delays on civil aviation safety risk is random. That is to say, the potential safety hazard which supervenes safety risk with hidden dangers is random. The concurrence probability is random, which is based on different delay rate and risk level.

(v) As a special safety risk, there is a causal relationship between different potential safety hazards to some extent. That is to say, as a potential safety hazard, it is uncertain and random that flight delays can induce and cause the variation of other dangers.

(vi) The influence of flight delays on civil aviation safety risk can be regarded as an increment risk, which belongs to the interaction between gradual accumulation and sudden surge of different random factors.

In conclusion, the source of random risk can be summarized as follows:

(i) the factors which induce flight delays and civil aviation safety are random;

(ii) the conditions which lead to flight delays and civil aviation safety are random;

(iii) the state of flight delays and civil aviation safety is random.

The randomness between factors, conditions, and states can be expressed in Figure 3.

\subsection{The Analysis on Change Rules of Civil Aviation Safety Risk Based on Flight Delays}

3.3.1. The Increase of Potential Safety Risks Leading to Civil Aviation Safety Risk. Flight delays are regarded as a kind of new safety risk. The characteristics of flight delays are that it exists when flight delay exists; otherwise, it disappears. 


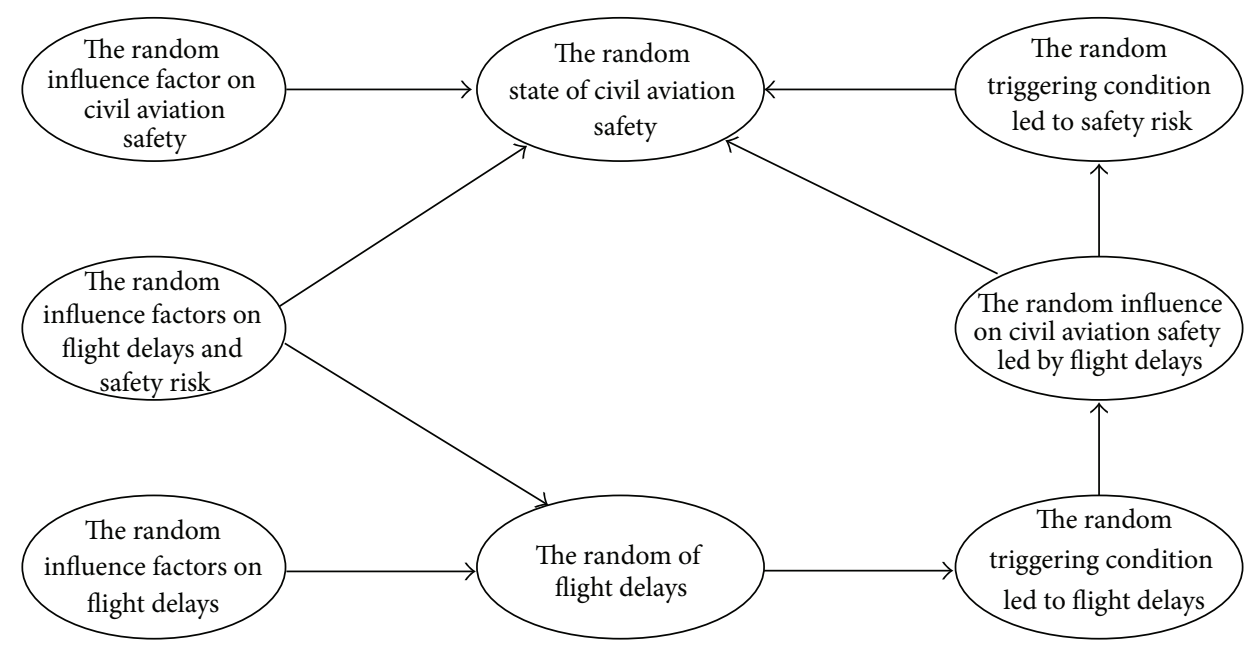

FIGURE 3: The diagram of random risk of civil aviation safety based on flight delay.

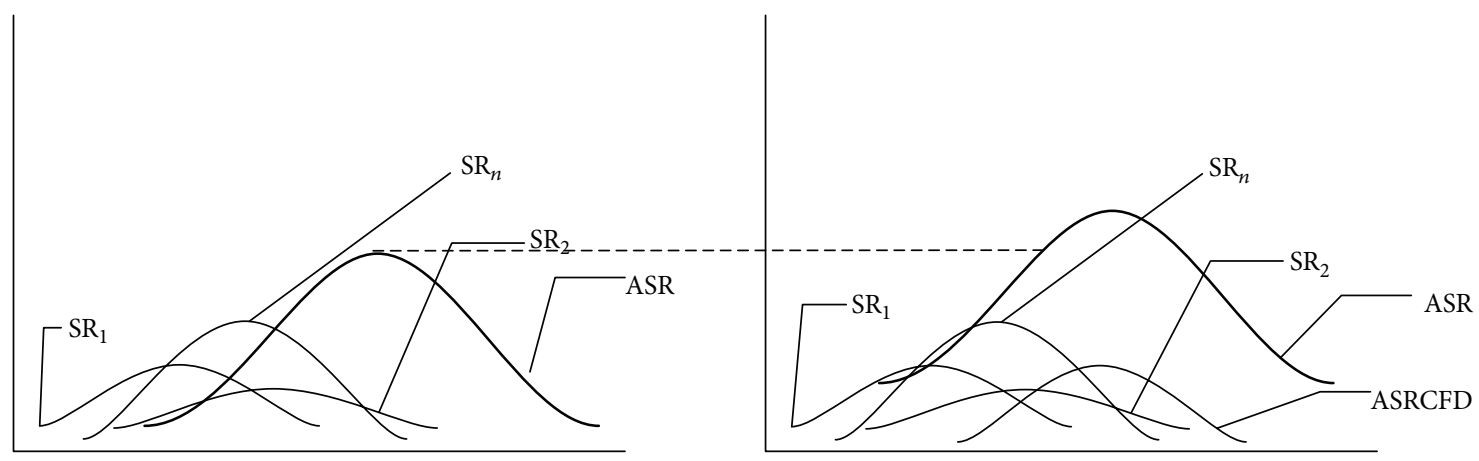

SR: safety risk

ASR: aviation safety risk

ASRCFD: aviation safety risk based on flight delays

FIGURE 4: The change rule of civil aviation safety risk considering flight delays as potential safety hazard.

Assuming that there are $n$ hidden risks ( $n$ risk sources) when there are no flight delays, there are $n+1$ hidden risks when flight delays occur. Even assuming that flight delays do not affect other hidden risks (e.g., civil aviation safety risk degree, which is caused by $n$ hidden dangers, does not vary), the $n+1$ hidden risks, including flight delay and safety risk induced by flight delays, will increase the degree of civil aviation safety risk.

The change rules of safety risk degree are shown in Figure 4 .

(i) Assuming that potential safety risk and flight delays are independent when delays flights are acting as a potential safety risk;

(ii) The reason that safety risk increases is that flight delays act as an independent potential safety risk;

(iii) Regular flight is a normal state, and flight delays are abnormal state. The existence of flight delays can lead to the increase of civil aviation risk inevitably; this is a systemic risk; (iv) As the change rule of potential safety risk in Figure 4, the paper has taken the randomness of risk change into account and assumes that the variation of risk obeys normal distribution.

3.3.2. The Common Causes of Safety Risk and Flight Delays Leading to Aviation Safety Risk. The factor which causes flight delays has an impact on civil aviation safety at the same time. Assuming that there are $n$ potential safety risks ( $n$ risk sources) when flight delays do not occur. The common factors not only lead to flight delays but also have an influence on the risk variation rules of primary potential safety risk $n$. That is to say, flight delays are not the direct reason which leads to the increase of civil aviation safety risk.

The mechanism of risk is shown in Figure 5.

According to Figure 5, the explanations include the following.

(i) The risk caused by flight delays which act as an independent potential safety hazard should not be taken into account. 


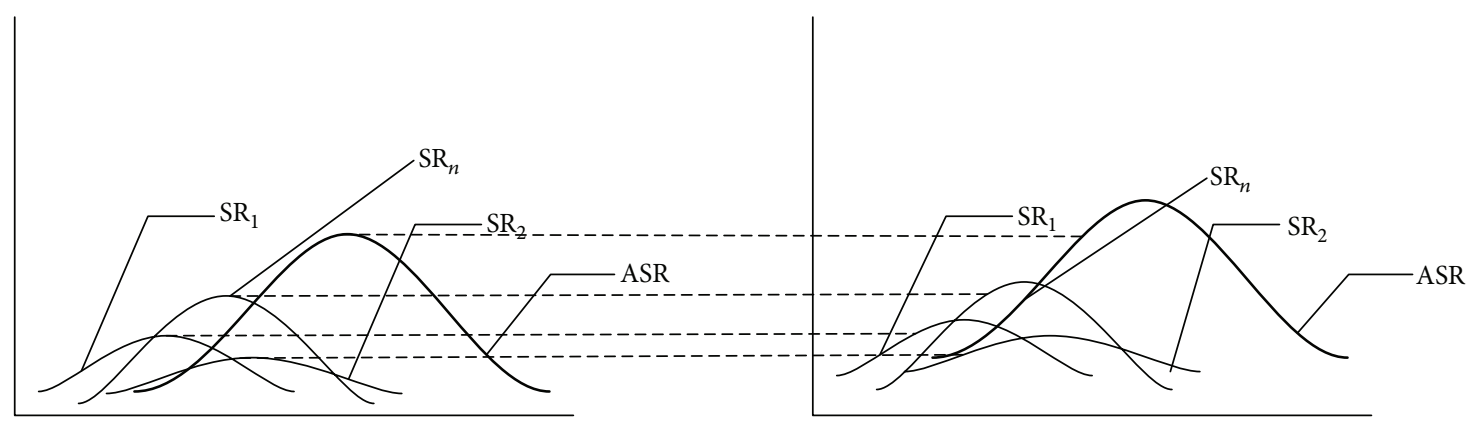

SR: safety risk

ASR: aviation safety risk

FIGURE 5: The change rule of civil aviation safety risk caused by the common causes of civil aviation and flight delays.

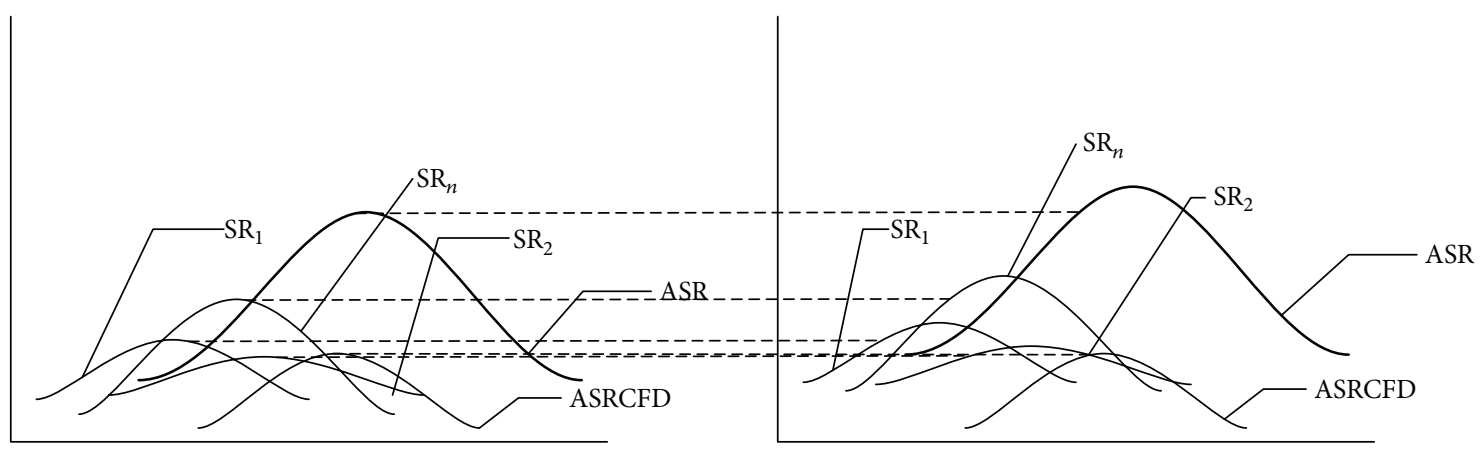

SR: safety risk

ASR: aviation safety risk

ASRCFD: aviation safety risk based on flight delays

FIGURE 6: The change rule of civil aviation safety risk under flight delay acting as inducement.

(ii) Figure 5 aims to analyze the influence on common factors of safety risk and flight delay on civil aviation safety risk. Compared with flight delay, the indications of safety risk (accident, accident symptom, and unsafe events) are much less sensitive to flight delay. That is to say, flight delay is the explicit index of common factors, and safety risk is shown as implicit index, usually.

(iii) The common factors of safety risk and flight delay lead to various change rules of risk on $n$ safety potential safety hazard. Although the degree, time, and variation rules are different, the entire trend is to increase the civil aviation risk.

(iv) Considering the randomness of risk change, the paper assumes that risk change obeys normal distribution and the civil aviation safety risk sources are composed of different risk sources.

3.3.3. Flight Delays Leading to the Increase of Civil Aviation Safety Risk Degree as an Inducement. In addition to the common factors, flight delay as an inducement will further have an effect on civil aviation. The actual safety risk is overlapped between the above two kinds of influence and the superposition may lead to a sound increase in safety risk degree objectively.

Flight delays acted which as inducement increased the risk degree of civil aviation safety, and the change rule of risk is showed in Figure 6.

From Figure 6, we can draw some conclusions.

(i) Flight delays as an inducement in Figure 6 directly have an impact on potential safety risk, which acted as the occurrence of flight delay and stimulates and triggers the change of safety risk degree.

(ii) As the change rules of different potential safety risk in Figure 4, the paper has taken the randomness of risk change into account and assumes that risk change obeys the normal distribution.

It is worth mentioning that the risk change rule of civil aviation safety in Figures 4 and 6 has been assumed to obey normal distribution. The assumption is from general knowledge about civil aviation safety risk, which is not carefully calculated. The detailed calculation about safety risk of civil aviation can be seen in Section 4 . 


\section{Risk Assessment Model of Civil Aviation Safety Based on BN}

4.1. Overview of $B N$. $B N$ is a directed acrylic graph for reasoning under uncertainty in which the nodes represent variables and are connected by means of directed arcs. The arcs denote dependencies or causal relationship between the link nodes and the conditional probability table assigned to the nodes determining how the linked nodes are dependent on each other [18].

Based on the conditional independency theorem and the chain rule, $\mathrm{BN}$ factorizes the joint probability distribution of a set of random variables $U=\left\{A_{1}, A_{2}, \ldots, A_{n}\right\}$ by considering local dependencies. The joint probability distribution can be decomposed as the product of the probabilities of the nodes given their immediate parents [19]:

$$
P(U)=\prod_{i=1}^{n} P\left(A_{i} \mid P a\left(A_{i}\right)\right)
$$

where $P(U)$ is the joint probability distribution of variables and $\mathrm{Pa}\left(A_{i}\right)$ is the parent set of variable $A_{i}$.

$\mathrm{BN}$ takes advantage of the Bayes theorem to update the probability of events given a new observation, called evidence $E$, to yield the posterior probability:

$$
P(U \mid E)=\frac{P(U, E)}{P(E)}=\frac{P(U, E)}{\sum_{U} P(U, E)} .
$$

Using (2) probability can be updated by versatile type of $E$.

$\mathrm{BN}$ is the method integrated by quality analysis and quantitative analysis, which can utilize many kinds of information. $\mathrm{BN}$ has the function of learning itself, backward reasoning, and inference in incomplete data sets. The above advantages make BN superior to other artificial intelligence methods in safety evaluation of civil aviation based on flight delays.

$\mathrm{BN}$ has been widely applied in engineering domain, such as risk analysis [20-22] and fault diagnosis [23, 24] and prediction $[25,26]$.

\subsection{The BN Model for Civil Aviation Safety Risk Assessment.} There are six steps in $\mathrm{BN}$ model to assess the civil aviation safety risk.

Step 1 (extract the key factors causing flight delays and civil aviation safety risk and determine the BN nodes). Determination of nodes is the foundation and key for determining the structure of BN. There are four categories of nodes to be taken into account. First, the common cause factors for flight delay and civil aviation safety should be taken into account. Second, the independent causing factors for flight delays and civil aviation safety should be taken into account. Third, the factors of flight delays which induced safety risk of civil aviation operation should be taken into account. Forth, the prorogation and spread of flight delay itself should be taken into account.

Step 2 (determine the risk assessment BN structure of civil aviation operation based on flight delays). The model should reflect the actual civil aviation safety. The nodes determined in Step 1 are connected with causality. So, the structure of $\mathrm{BN}$ consisted of causality chain, which can be required from logic analysis and expert experience. The determination of $\mathrm{BN}$ structure is the qualitative modeling in $\mathrm{BN}$. The analyses of Sections 2 and 3 are used to build the BN structure of civil aviation safety risk.

Step 3 (instantiate the $\mathrm{BN}$ with probabilities). First, prior probabilities are assigned to the root nodes in the $\mathrm{BN}$ for civil aviation safety risk assessment. To root node $X_{i}, P\left(x_{i}=\right.$ $R)$ and $P\left(x_{i}=N\right)$ represent risk probability and normal probability of node $x_{i}$, respectively, and $P\left(x_{i}=R\right)+P\left(x_{i}=\right.$ $N)=1$.

Next, conditional probabilities are assigned to other nodes. To a child node with $n$ parents, $2^{n}$ conditional probabilities should be assigned. It is difficult to collect much information in actual civil aviation operation and management. Experts also have some difficulty in providing so much information. To overcome the problem, it is necessary to study the $\mathrm{BN}$ for civil aviation safety risk assessment for simplifying the conditional probabilities assignment.

Step 4 (learn BN structure). There are two contents in $\mathrm{BN}$ structure learning. One way is to decide $\mathrm{BN}$ structure by data reasoning. The other way is to verify structure of $\mathrm{BN}$ and delete weak connections between nodes by data if the initial structure of $\mathrm{BN}$ has been known. In the paper, the initial $\mathrm{BN}$ structure of civil aviation safety risk assessment has been decided based on the study of Sections 2 and 3. The emphasis of $\mathrm{BN}$ on civil aviation safety risk assessment is to simplify BN structure. Markov blanket is selected to $\mathrm{BN}$ in civil aviation operation risk.

Step 5 (learn BN parameters). Bayes method is the theory basis for BN. Bayes method uses prior density and posterior density to learn and assess parameters. $\mathrm{BN}$ also uses the above process to learn parameters after collecting and accumulating relevant data. In the practical application, the parameters learning of $\mathrm{BN}$ also use conjugate prior to simplify parameters learning.

Step 6 (infer BN). Civil aviation safety risk can be assessed by $\mathrm{BN}$ if the $\mathrm{BN}$ structure has been known and the nodes have been assigned. In the paper, GeNIe has been selected to compute because of large amount of calculation.

4.3. The BN Structure of Civil Aviation Safety Risk Assessment. The analysis on designing BN structure of civil aviation safety risk assessment bases on flight delay.

(i) Civil aviation safety system is the complex large system, which includes flight subsystem, maintenance subsystem, air traffic control subsystem, and airport subsystem. These subsystems are affected by flight delays in different degree. These subsystems also have an impact on civil aviation safety risk in different mode. So, it is necessary to use independent analysis for different subsystems. 
TABLE 1: The description of node characteristics.

\begin{tabular}{lc}
\hline Meaning & Abbreviation \\
\hline Weather & WE \\
Flow control & FC \\
Maintenance and engineering & ME \\
Airplane plan & AP \\
Airport design & AD \\
Airport order & AO \\
Scheduled flight time & SFT \\
ATC safety risk & ATSR \\
Airline safety risk & ALSR \\
Airport safety risk & APSR \\
Maintenance safety risk & MSR \\
Airport size & AS \\
Food supply & FS \\
Other reason & OR \\
Traveler & TR \\
Aircraft type & AT \\
Flight delay & FD \\
Station flight delay & SFD \\
Flight delay rate & FDR \\
Risk rate of aviation operation safety & AOSRR \\
Safety operation risk based on flight delay & SRBFD \\
ATC safety hidden danger & ATSHD \\
Airline safety hidden danger & ALSHD \\
Airport safety hidden danger & APSHD \\
Maintenance safety hidden danger & MSHD \\
Prohibited fly & PF \\
Air crew & AC \\
Trant check & SC \\
\hline
\end{tabular}

(ii) Flight subsystem, maintenance subsystem, air traffic control subsystem, and airport subsystem are all affected by person, machine, environment, and management. To avoid large BN structure, modular structure has been introduced in the paper. The nodes of hidden risk and safety risk have been designed in different subsystems, the value of which can be calculated by $\mathrm{BN}$ inference.

(iii) In order to analyze the flight delays which propagated safety risk, the flight delays modeling should be emphasized, which include influence factors such as flight delays, flight delays propagation, flight delays rate, and risk assessment based on flight delays.

The initial risk assessment $\mathrm{BN}$ of civil aviation safety is shown in Figure 7. The meaning of nodes in Figure 7 is shown in Table 1.

\subsection{BN Learning for Civil Aviation Safety Risk Assessment}

4.4.1. BN Structure Learning of Civil Aviation Safety Risk Assessment. A novel idea of $\mathrm{BN}$ of significant feature selection is the Markov blanket (MB). In order to simplify $\mathrm{BN}$ structure of civil aviation safety risk assessment, Markov blanket is selected in $\mathrm{BN}$ structure learning.

$\mathrm{MB}$ is defined as the set of input features because all the other features are probabilistically independent of target features. In a Bayesian network, the Markov blanket [27] of a node $X_{i}$ (which can be represented as $\mathrm{MB}\left(X_{i}\right)$ in short) is the set of nodes which is composed of its parent nodes, its child nodes, and parent nodes of its child nodes. The important property of Markov blanket is that Markov blanket of variable $X_{i}$ is the set of nodes which makes $X_{i}$ independent in the network. That is to say, the nodes belonging to the Markov blanket of $X_{i}$ are the most relevant to $X_{i}$,

$$
P\left(X_{i} \mid X_{1}, \ldots, X_{i-1}, X_{i+1}, \ldots, X_{n}\right)=P\left(X_{i} \mid \operatorname{MB}\left(X_{i}\right)\right) .
$$

The probability of the target node is influenced only by its Markov blanket. Therefore, MB (target node) is the most informative and relevant to risk assessment of civil aviation safety.

For example,

$P($ AOSRR | MB (AOSRR))

$=P($ AOSRR $\mid$ SFD, WE, FFD, ALSR, APSR, ARBR, MSR)

$=\frac{P(\mathrm{AOSSR}, \mathrm{SFD}, \mathrm{WE}, \mathrm{FFD}, \mathrm{ALSR}, \mathrm{APSR}, \mathrm{ARBR}, \mathrm{MSR})}{P(\mathrm{SFD}, \mathrm{WE}, \mathrm{FFD}, \mathrm{ALSR}, \mathrm{APSR}, \mathrm{ARBR}, \mathrm{MSR})}$

$=\frac{\prod_{X_{i} \in\{\text { SFD,WE,FFD,ALSR,APSR,ARBR,MSR }\}} P\left(X_{i} \mid \pi\left(X_{i}\right)\right)}{P(\text { SFD, WE, FFD, ALSR, APSR, ARBR, MSR })}$

$=P(\operatorname{AOSRR} \mid \pi(\mathrm{AOSRR}))$

$\prod_{X_{i} \in \text { Children(AOSRR) }} P\left(X_{i} \mid \pi\left(X_{i}\right)\right)$

$$
\begin{aligned}
& \prod_{X_{j} \notin \text { AOSRR } X_{j} \notin \text { Children(AOSRR) }} P\left(X_{j} \mid \pi\left(X_{j}\right)\right) \\
& \times(P(\text { SFD, WE, FFD, ALSR, APSR, ARBR, MSR }))^{-1} .
\end{aligned}
$$

4.4.2. BN Parameters Learning for Aviation Safety Risk Assessment. In the $\mathrm{BN}$ of civil aviation safety risk assessment, assuming that node has a multinomial distribution, we can parameterize it with the parameter vector $\theta_{1}, \theta_{2}, \ldots, \theta_{S}$, where $S$ is the number of states of variable $x$ and $\theta(k)=P\left(x=x_{k} \mid\right.$ $p)$, for $1 \leq k \leq S$.

(1) $\theta$ possess the Dirichlet distribution

$$
\theta \sim D\left[\alpha_{1}, \alpha_{2}, \ldots, \alpha_{S}\right]
$$

$\alpha_{i}>0 ; i=1, \ldots, S$, and $\sum_{i=1}^{S} \theta_{i}=1$, we can act $\alpha_{i}$ as presenting counts of past cases which are stored as a summary of experience. 


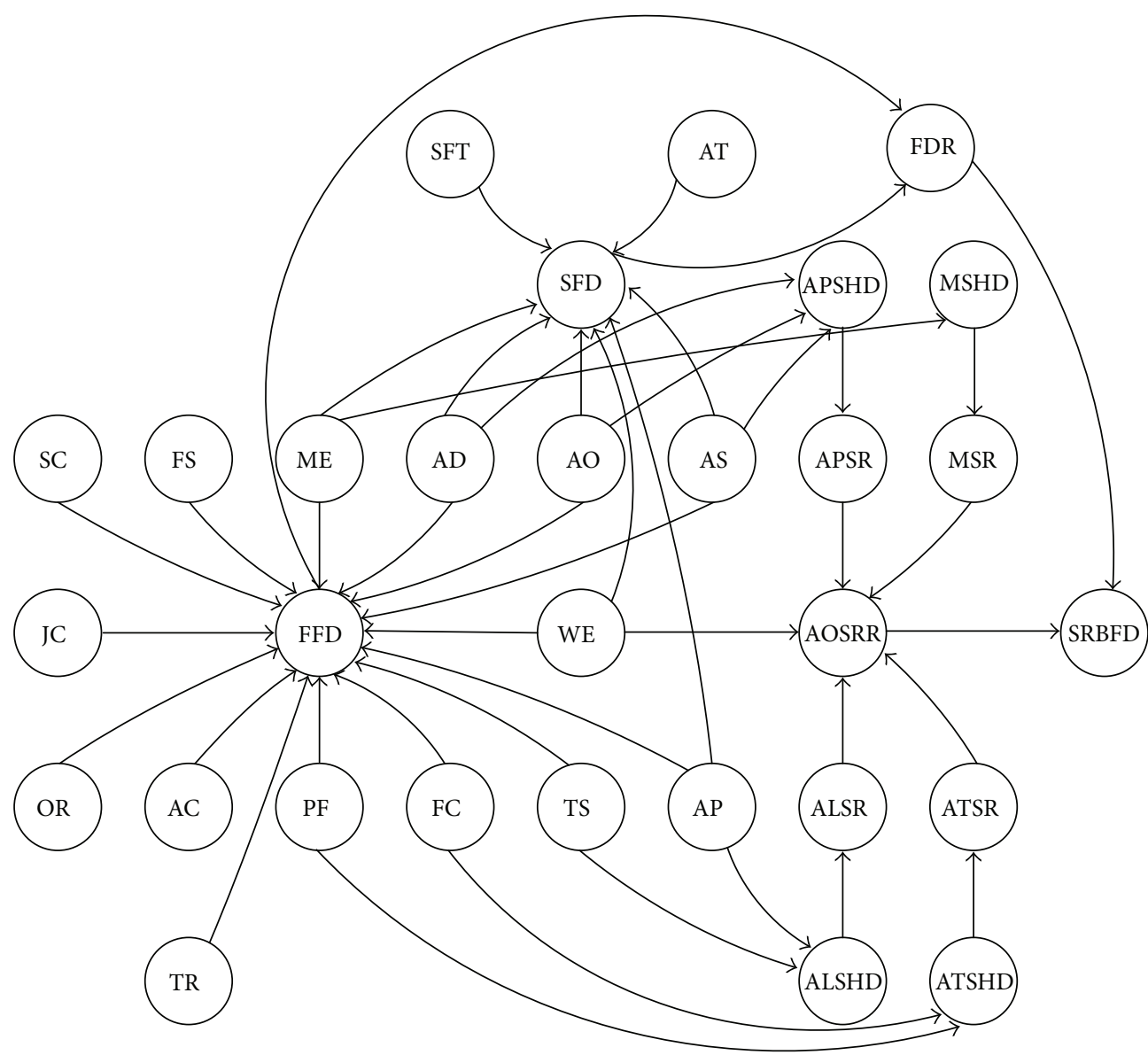

FIGURE 7: BN structure of civil aviation safety operation assessment based on flight delay.

(2) Let $\alpha_{0}=\sum_{i=1}^{S} \alpha_{i}$ and let expected value, variance, and covariance of Dirichlet distribution be defined as follows:

$$
\begin{gathered}
E\left(\theta_{i}\right)=\frac{\alpha_{i}}{\alpha_{0}}, \\
\operatorname{Var}\left(\theta_{i}\right)=\frac{\alpha_{i}\left(\alpha_{0}-\alpha_{i}\right)}{\alpha_{0}^{2}\left(\alpha_{0}+1\right)}, \\
\operatorname{Cov}\left(\theta_{i}\right)=-\frac{\alpha_{i} \alpha_{j}}{\alpha_{0}^{2}\left(\alpha_{0}+1\right)} .
\end{gathered}
$$

We assume that $\theta$ prior distribution is Dirichlet distribution; then posterior distribution is also Dirichlet distribution; they are conjunction distribution.

When the samples are $N=\left(N_{1}, N_{2}, \ldots, N_{S}\right)$, the posterior expected value of $\theta_{k}$ with $k=1,2, \ldots, S$ is given by

$$
E\left(\theta_{i} \mid \alpha, N\right)=\frac{\alpha_{i}+N_{i}}{\sum_{i=1}^{S}\left(\alpha_{i}+N_{i}\right)}
$$

\section{Examples}

The paper selects an airline company in China to verify the model in the paper. The relevant data in Figure 7 were collected in 2012.
The data comes from the following sources.

(i) Some data is obtained by statistics analysis, such as FC node. The FC percentage can be calculated by statistics data.

(ii) Some data is difficult to collect directly, which is inferred by historical data and expert information.

(iii) Some data is obtained by other ways and analysis methods, such as hinder risk of airport. Because computation on hinder risk of airport is very complex, the result of airport safety audit of last year can be used to reduce the computation difficulty.

In the paper, calculation of time interval is a month. The data of 2011 can be used as prior information. After collecting the data of January 2012, the marginal posterior probability of each node in the risk assessment BN of civil aviation safety can be calculated for January 2012. Structure learning and parameter learning of $\mathrm{BN}$ are in the whole calculation process. Then posterior probability of each node in January 2012 can be used as the prior information of February 2012. After collecting data of February 2012, the marginal posterior probability of each node in the risk assessment $\mathrm{BN}$ of civil aviation safety can be calculated for February 2012. In the same way, the posterior marginal probability of each node in every month of 2012 can be calculated. 


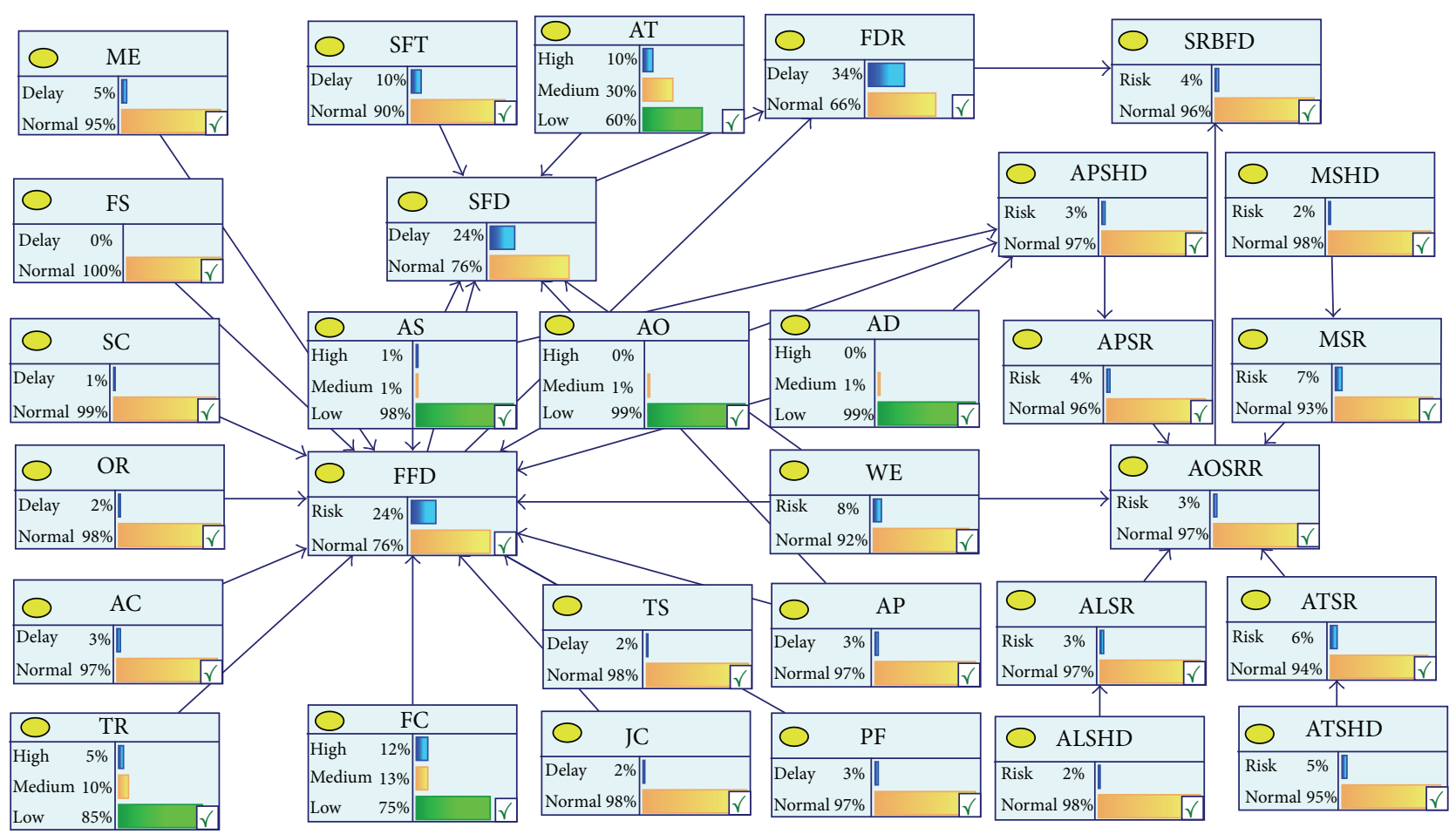

FIGURE 8: Posterior marginal probability of each node in January 2012.

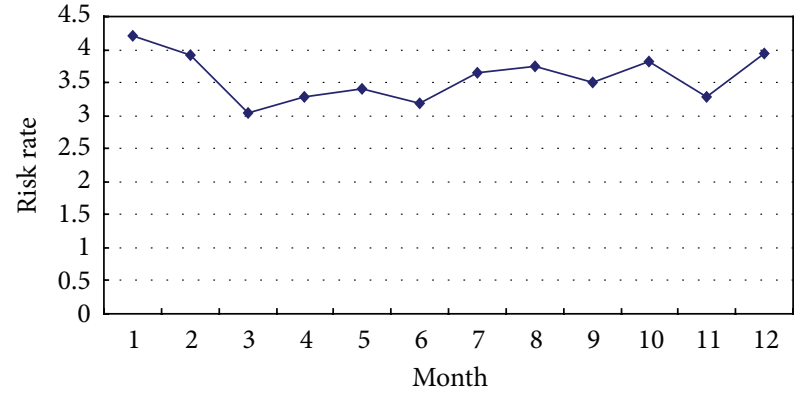

FIGURE 9: The safety risk probability of civil aviation operation in 2012.

For example, the posterior margin probability of nodes in January 2012 is shown in Figure 8

From Figure $8, P($ AOSRR $=R)=2.960 \%$ represents the safety risk of civil aviation operation system itself. $P$ $($ SRBFD $=$ Risk $)=4.241 \%$ represents the safety risk of civil aviation system based on flight delays. From the result in Figure 8, fight delays substantially increase in the safety risk probability of civil aviation, which increase the safety risk probability by $43.77 \%$.

The safety risk probability of civil aviation for each month in 2012 has been calculated in Figure 9. Flight delays probability of civil aviation for each month in 2012 has been calculated in Figure 10. From Figures 9 and 10, the results demonstrate that there is a closer relationship between flight delays and safety risk of civil aviation; the trend is basically

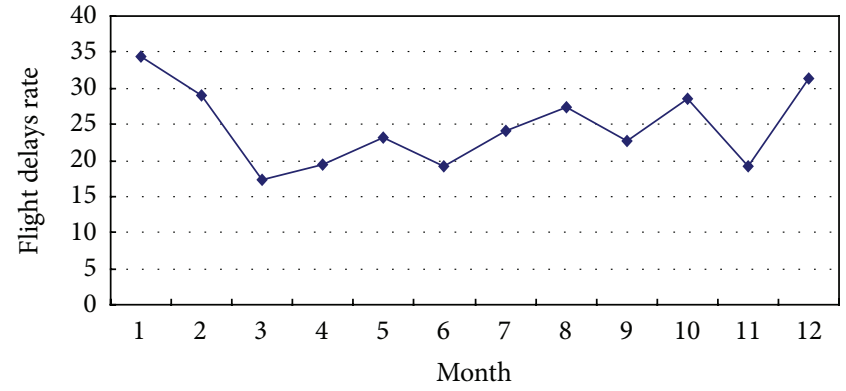

FIGURE 10: The civil aviation safety risk rate based on flight delay in 2012.

the same. When flight delays increase dramatically, safety risk probability greatly increases. So it is necessary to pay close attention to safety risk of civil aviation operation caused by flight delays.

The following conclusions can be drawn by the above results to strength the safety management of civil aviation in China.

(i) Fight delays are not related to the service quality and operation efficiency of civil aviation but do have a relatively great impact on civil aviation safety. The trend of flight delay rate and civil aviation risk rate is the same, which illustrates that flight delays have effect on civil aviation safety. 
(ii) Partial factors which caused flight delays are controllable. The other factors which caused flight delays are not only uncontrollable but also unpredictable, which determines the difficulty of managing flight delays in China. With the development of Chinese civil aviation, the number of arrival and departure flights grows rapidly. If the managers cannot manage the flight delays effectively, the rate of flight delays will increase and the effect of flight delays will spread, which will lead to huge pressure on civil aviation safety.

(iii) It is an efficient way for strengthening the civil aviation by details management in China. The element state change in civil aviation safety system should be avoided and improved the safety management level of civil aviation in China.

\section{Conclusions}

The paper analyzes the relationship between flight delays and civil aviation safety risk. The problem of flight delays accompanied and propagated with civil aviation safety risk has been presented. The problem of flight delays leading to propagation and superposition of civil aviation safety risk has been analyzed. The $\mathrm{BN}$ has been used to model safety risk assessment of civil aviation safety. The example of some airlines in China has demonstrated the effectiveness and correctness of method. The countermeasures and suggestions have been put forward to solve the problems of Chinese civil aviation safety in the process of rapid development.

\section{Acknowledgments}

This work was supported by the National Natural Science Foundation and Aviation Fund of China (no. 60879001 and no. U1233115) and the Qinglan Project.

\section{References}

[1] M. Sachon and E. Paté-Cornell, "Delays and safety in airline maintenance," Reliability Engineering and System Safety, vol. 67, no. 3, pp. 301-309, 2000.

[2] M. V. McCrea, H. D. Sherali, and A. A. Trani, "A probabilistic framework for weather-based rerouting and delay estimations within an Airspace Planning model," Transportation Research C, vol. 16, no. 4, pp. 410-431, 2008.

[3] P. Zheng, S.-J. Hu, and C. Zhang, "Chaotic phenomena and quantitative analysis of aftereffect delay spread time on flights," Journal of Transportation Systems Engineering and Information Technology, vol. 10, no. 4, pp. 68-72, 2010.

[4] J.-T. Wong and S.-C. Tsai, "A survival model for flight delay propagation," Journal of Air Transport Management, vol. 23, pp. 5-11, 2012.

[5] S. Yan and C.-H. Tang, "A heuristic approach for airport gate assignments for stochastic flight delays," European Journal of Operational Research, vol. 180, no. 2, pp. 547-567, 2007.

[6] N. Pyrgiotis, K. M. Malone, and A. Odoni, "Modelling delay propagation within an airport network," Transportation Research C, vol. 27, pp. 60-75, 2013.
[7] G. Santos and M. Robin, "Determinants of delays at European airports," Transportation Research B, vol. 44, no. 3, pp. 392-403, 2010.

[8] J.-C. Ferrer, P. Rocha e Oliveira, and A. Parasuraman, "The behavioral consequences of repeated flight delays," Journal of Air Transport Management, vol. 20, pp. 35-38, 2012.

[9] H.-J. Shyur, "A quantitative model for aviation safety risk assessment," Computers and Industrial Engineering, vol. 54, no. 1, pp. 34-44, 2008.

[10] M. Janic, "An assessment of risk and safety in civil aviation," Journal of Air Transport Management, vol. 6, no. 1, pp. 43-50, 2000.

[11] C.-C. Chen, J. Chen, and P.-C. Lin, "Identification of significant threats and errors affecting aviation safety in Taiwan using the analytical hierarchy process," Journal of Air Transport Management, vol. 15, no. 5, pp. 261-263, 2009.

[12] K. B. Marais and M. R. Robichaud, "Analysis of trends in aviation maintenance risk: an empirical approach," Reliability Engineering and System Safety, vol. 106, pp. 104-118, 2012.

[13] P. Brooker, "Experts, Bayesian belief networks, rare events and aviation risk estimates," Safety Science, vol. 49, no. 8-9, pp. 1142$1155,2011$.

[14] M. Hadjimichael, "A fuzzy expert system for aviation risk assessment," Expert Systems with Applications, vol. 36, no. 3, pp. 6512-6519, 2009.

[15] W.-K. Lee, "Risk assessment modeling in aviation safety management," Journal of Air Transport Management, vol. 12, no. 5, pp. 267-273, 2006.

[16] A. Rose, "Measuring operational safety in aviation," Aircraft Engineering and Aerospace Technology, vol. 78, no. 1, pp. 26-31, 2006.

[17] C. V. Oster Jr., J. S. Strong, and C. Kurt Zorn, "Analyzing aviation safety: problems, challenges, opportunities," Research in Transportation Economics, vol. 43, no. 1, pp. 148-164, 2013.

[18] F. V. Jensen and T. D. Nielson, Bayesian Networks and Decision Graphs, Springer, New York, NY, USA, 2nd edition, 2007.

[19] J. Cheng, R. Greiner, J. Kelly, D. Bell, and W. Liu, "Learning Bayesian networks from data: an information-theory based approach," Artificial Intelligence, vol. 137, no. 1-2, pp. 43-90, 2002.

[20] P. Weber, G. Medina-Oliva, C. Simon, and B. Iung, "Overview on Bayesian networks applications for dependability, risk analysis and maintenance areas," Engineering Applications of Artificial Intelligence, vol. 25, no. 4, pp. 671-682, 2012.

[21] D. Zhang, X. P. Yan, Z. L. Yang et al., "Application of Bayesian belief networks in navigation risk estimation of the Yangtz river," Reliability Engineering and System Safety, vol. 118, pp. 93105, 2013.

[22] M. J. Akhtar and I. B. Utne, "Human fatigue's effect on the risk of maritime groundings-a Bayesian network modeling approach," Safety Science, vol. 62, pp. 427-440, 2014.

[23] Y. Zhao, F. Xiao, and S. W. Wang, "An intelligent chiller fault detection and diagnosis methodology using Bayesian belief network," Energy and Buildings, vol. 57, pp. 278-288, 2013.

[24] C. H. Lo, Y. K. Wong, and A. B. Rad, "Bond graph based Bayesian network for fault diagnosis," Applied Soft Computing Journal, vol. 11, no. 1, pp. 1208-1212, 2011.

[25] S. Y. Sohn and A. S. Lee, "Bayesian network analysis for the dynamic prediction of early stage entrepreneurial activity index," Expert Systems with Applications, vol. 40, no. 10, pp. 4003-4009, 2013. 
[26] Y. F. Xu, J. Choi, S. Dass et al., "Efficient Bayesian spatial prediction with mobile sensor networks using Gaussian Markov random fields," Automatica, vol. 49, no. 12, pp. 3520-3530, 2013.

[27] J. Pearl, Probabilistic Reasoning in Intelligent Systems, Morgan Kaufmann, 1988. 


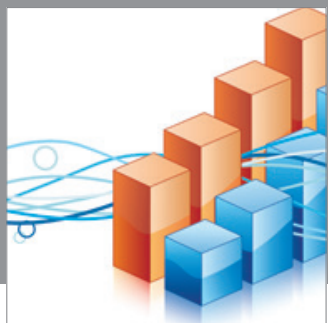

Advances in

Operations Research

mansans

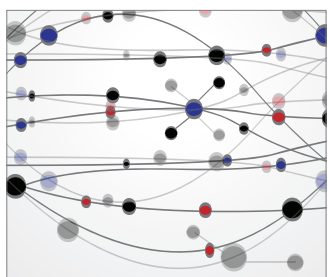

The Scientific World Journal
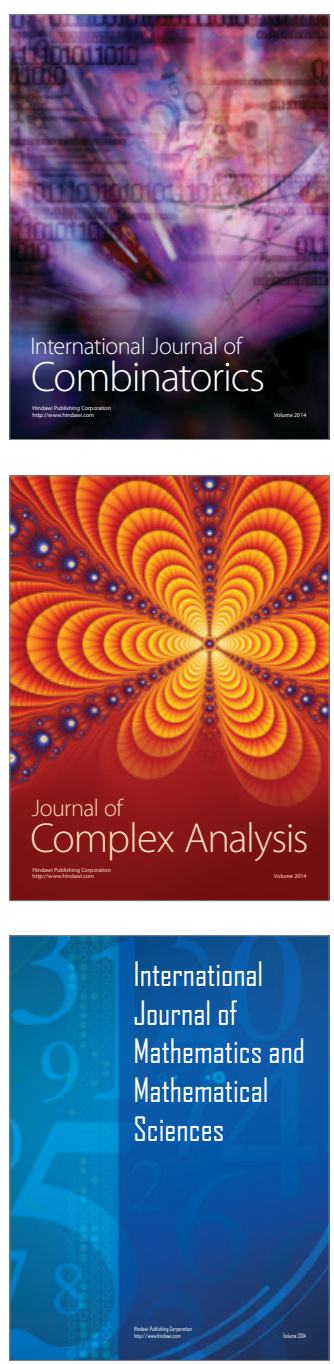
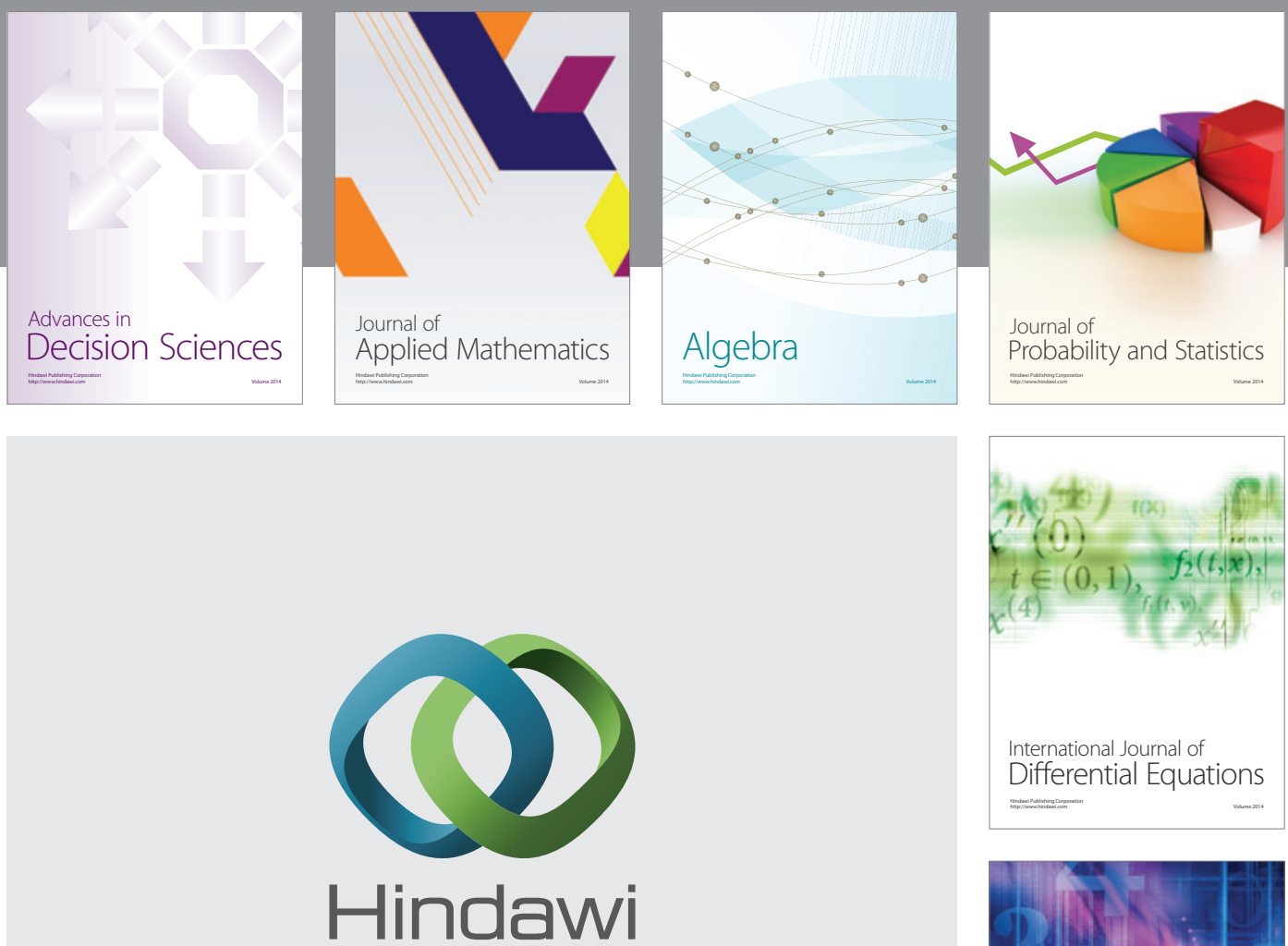

Submit your manuscripts at http://www.hindawi.com
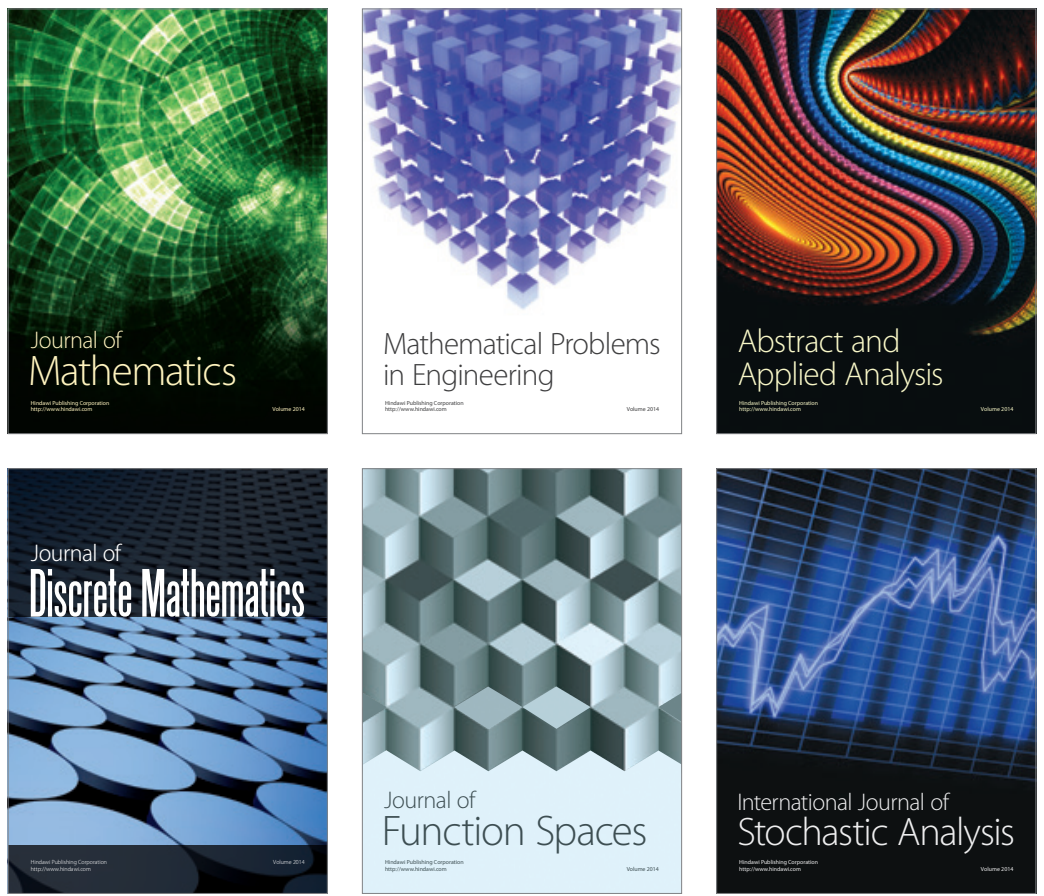

Journal of

Function Spaces

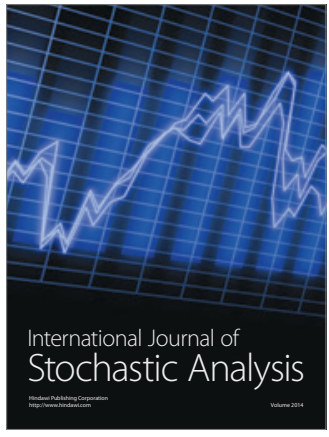

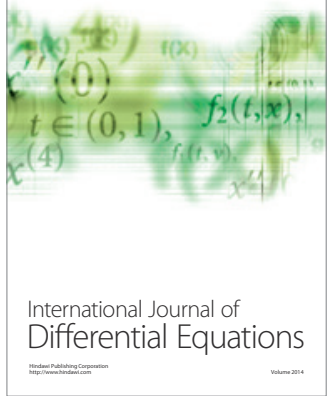
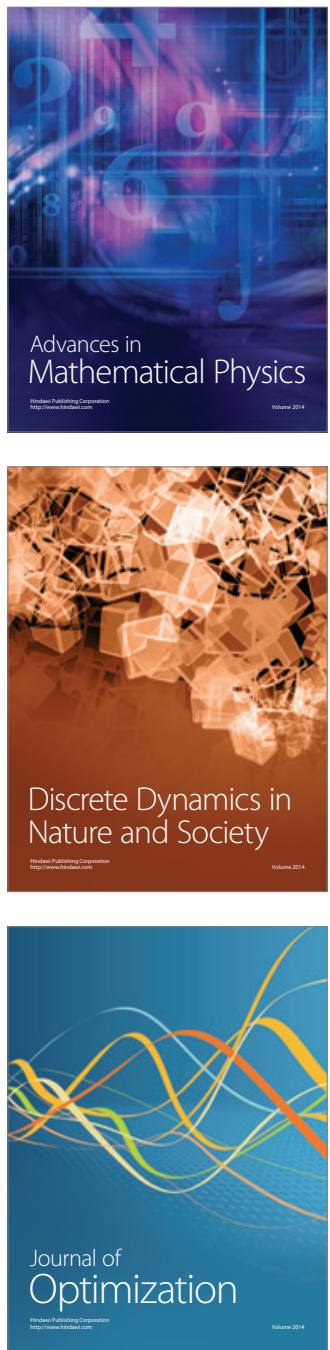